\title{
Exploratory Research on Exploitation Potentials for Forest-biomass based on the Social Development
}

\author{
Zhen Liu ${ }^{1,2}$ \\ 1. Marketing Department \\ Chongqing Real Estate College \\ Chongqing, China \\ 2. Institute of Management \\ Chongqing University of Technology \\ Chongqing, China; \\ Yang Weiqiang* \\ Institute of Management \\ Chongqing University of Technology \\ Chongqing, China; \\ justforyang@foxmail.com \\ * Corresponding Author
}

\author{
Gangqing $\mathrm{Pu}$ \\ Institute of Management \\ Chongqing University of Technology \\ Chongqing, China;
}

\begin{abstract}
With rising energy prices and growing pollution, biomass energy development has become a new research focus. The assessment potential for traditional forest biomass was only based on a single "natural" perspective, while ignoring the greatest social factors in the development of biomass energy cycle. Therefore, the research will consider some basic social factors, such as demographic change, food demand, food production and other factors, for the development of biomass energy. The factors based on different scenarios will be predicted by statistical methods, scenario analysis, econometric analysis method, etc; based on predictions, forest areas for "returning farmland to forest" or "returning forest to farmland" will be calculated ,thus calculating the corresponding potentials for forest biomass energy development. The research concluded that the potential for biomass energy will be up to $\mathbf{1 . 1 1 6}$ billion,1.272billion,1.412billion respectively in 2020,2030 , 2040 , 2050.,equaling to $6.73,7.79,9.04$ million tons of standard coal; When considering the social factors, a total of 108 biomass energy development potential results are obtained; the highest potential can reach $\mathbf{1 . 1 1 6}$ billion $\mathbf{1 . 2 7 2}$ billion 1.412 billion 1.551 billion tons respectively, equaling to $7.48,8.52,9.46,10.39$ million tons of standard coal; the lowest can reach 0.965 billion,1.105 billion,1.239 billion,1.376 billion respectively, equaling to $6.47,7.40,8.30,9.22$ million tons of coal. It concludes that before and middle social factors have a great impact on biomass energy development potential, which may cause potential decline. But in the long run, the social factors will increase the development potential for biomass energy.
\end{abstract}

Keywords-Biomass;Forestry;Agriculture;Scenario Analysl; social development ;)

\section{INTRODUCTION}

Because of the impact of energy prices and ecological problems, many national governments, organizations have begun to shift attention from the traditional energy to renewable, light pollution and relatively low price of biomass energy. According to forecast, with the deepening development of biomass energy in 2050 will become the most important source of energy, providing 40 percent of the world's fuel and 60 percent of the electricity. ${ }^{[1]}$ Forests are an important source of biomass energy, renewable energy sources forest biomass plays a great part (accounting for approximately $90 \%$ of global fixed energy), ${ }^{[2]}$ In the world, the barren wasteland area, about 100 million $\mathrm{hm}^{2}$, At least $20 \mathrm{t}$ of biomass resources can be developed, equal to $10 \mathrm{t}$ standard coal ${ }^{[3]}$. China as a big country of forestry, So far in 2014, forest area reached 208 million hectares, biomass energy development potential is very great. However, the development of forestry biomass want better, effective assessment of forestry biomass energy development potential is one of the very important prerequisite. At present, China's forestry biomass energy development potential assessment is mainly from forest area, stock volume and others unilateral static perspectives. Such as Lv Wen (2005), Qian Nengzhi (2007), Zhou Xiao (2009) All of them calculate the potential of China, Europe, Yunnan Province's biomass energy are from the perspective of the total amount of forest resources reserves This does not relate to biomass development cycle affecting the most important social factor, ${ }^{[4-6]}$ and for our part, Forest biomass energy development is bound to be social factors of population, land, food needs, food production and other effects, but the related research is less.

Now, the premise of land use in China is food security, this means that any other uses of land development and utilization are under the premise of ensuring food security. In terms of population, according to the "national population development strategy research report" ${ }^{[7]}$ In 2033 our country will reach its peak population of about 1.5 billion people, and then there will be a population decline, "Changes and adjustments demographic situation of population policy (China Development Report 2011/2012)" [8] also generally agree with this view; "China Population Prospects 2015-2080' using the cohort method and according to Taiwan, South Korea as a forecast of the 
future population growth will be slower, the peak of the population can not reach 15 million and aging serious. ${ }^{[9-10]}$ In terms of food needs, Shichang Liang (2013), Deng Jing (2003), Tang Huajun (2012) by statistical methods, linear programming, literature data search and nutritional perspective concluded that China's per capita ration demand will maintain a relatively stable state, while other food needs to maintain a steady slow growth trend. ${ }^{[11-13]}$. According rubles (2005), Chen Fangyuan (2006), Zhu Qing (2003), Ren BingXue (2009), Zhang Yongen (2009) and other scholars through linear programming, gray combined forecasting, and statistical methods to draw huge potential for our future food yield and the growth rate has remained higher than demand growth. ${ }^{[14-18]}$ Therefore, the future demand for arable land area will be greatly reduced, and when this situation occurs after the development of other ways of arable land will become a new topic. If these new land to develop biomass energy, the biomass potential can be raised.

So the study will be based on the actual situation in China, and according to forest land area and food taking into account the dynamic changes caused by social factors to forecast the future development potential of forestry biomass energy, and make the guidance for the development and utilization of biomass energy.

\section{PREDICTION OF BIOMASS ENERGY DEVELOPMENT}

\section{A. Calculation process of biomass development potential}

Starting from the premise of ensuring food security and the current forestry planning, according to the future demand for grain and grain yield per hectare, According to the future demand for grain and grain yield getting the actual demand for farming area and excess area; According to the forecast results, "returning farmland to forest" or "returning farmland to forest" and get the forest area under the relevant circumstances ,and calculated to obtain the final biomass energy development potential. Finally, compare the impact of different scenarios on biomass energy development and propose the development proposals for the future. Specific procedures are as follows:

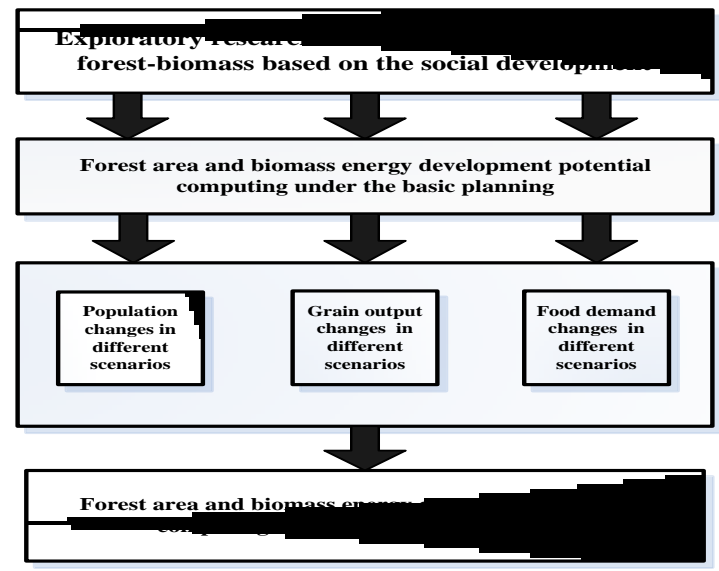

Figure 1. Calculation flow chart
B. Calculation model of biomass energy development potential based on social development

1) The total biomass potential prediction model given by equation (1)

$\mathcal{Q}_{n}^{i}=\sum_{r=1}^{5}\left(\mathcal{A}_{\mathrm{n}}^{i} \times \mathcal{M}_{r}^{i} \times \phi^{r}\right)+\mathcal{D}_{n}+\mathcal{Z}$

$\mathcal{Q}_{n}^{i}$ represents in the first $\mathrm{n}$ years, biomass energy development potential of the development model of $i ; \mathcal{A}_{\mathrm{n}}^{i}$ represents forest area in the first $n$ years of the model of $i ; \mathcal{M}_{r}^{i}$ represents the area ratio of the I forest in the first $n$ years of development mode of $i ; \phi^{r}$ represents the $r$ biomass forest exploitation coefficient; $\mathcal{A}_{\mathrm{n}}^{i} \times \mathcal{M}_{r}^{i} \times \alpha^{r}$ represents the quality of the remainder of the forest in the $i$ modes; $\mathcal{D}_{n}$ represents the quality of the remaining material for cutting, building and processing in the first $n$ years. $Z$ represents the output constant of miscellaneous remainder, It includes: forest fire road construction, clearing fire tree, dead tree pests, all around, scattered rusty Tending residue, urban greening update residue, waste wood and other residues, planting seedlings branches off the residue. Because these specific amount is not easy to count and the amount of less, so choose to cite Zhang Xiliang (2008) calculated $2.35 \mathrm{t}$.

2) Forest area model given by Eq. (2)

$$
\mathcal{A}_{n}=\mathcal{L}_{n}-\left(\mathcal{S}_{n}-\mathcal{S}_{n-1}\right)
$$

$\mathcal{A}_{n}$ represents the dynamic forest area in the first $\mathrm{n}$ years; $\mathcal{L}_{n}$ represents the planning of forest area in the first $n$ years; $\mathcal{S}_{n}$ represents the area of food in the first $n$ years; $\mathcal{S}_{n-1}$ represents the area of food in the first n-1 years;

3) Food area model given by equation (3)

$$
\delta_{n}=\varepsilon_{n} / \mathcal{u}_{n}
$$

$\mathcal{S}_{n}$ represents of the food needs of arable land in the first $n$ year; $\mathcal{E}_{n}$ represents the total grain demand in the first $n$ year; $\mathcal{U}_{n}$ represents grain yield in the first $n$ year .

4) Food demand model is given by Eq. (4)

$$
\mathcal{E}_{n}=\left(\mathcal{P}_{n} \times q+\mathcal{Q}_{n}\right) \times \mathcal{O} \times\left(1+\mathcal{H}_{n}\right)
$$

$\mathcal{E}_{n}$ represents total grain demand in the first $n$ years; $\mathcal{P}_{n}$ represents the total population in the first $n$ year; $\mathcal{Q}_{n}$ represents the industrial food demand in different years; $\mathcal{O}$ represents a proportional constant demand for seed grain; $\mathcal{H}_{n}$ representing different food consumption ratio in the different year.

5) The total population of the model is given by Eq. (5)

$$
\mathcal{P}_{t+1}=\mathcal{P}_{t} \times\left(\mathcal{B}_{t}-\mathcal{D}_{t}\right)+\mathcal{M}_{t}
$$

$\mathcal{P}_{t} 、 \mathcal{B}_{t} 、 \mathcal{D}_{t}, \mathcal{M}_{t}$ represent the population in the first $t$ year, the number of births, deaths and net immigration number., and because our current immigration population is too low, negligible. $\mathcal{B}_{t}=$ the number of women of childbearing age $*$ birth rate, $\mathcal{D}_{t}=$ $\sum$ "each age population $*$ the probability of death (the death rate of life table data of life insurance in Taiwan area).

6) The grain yield model is given by Eq. (6)

$$
\mathcal{U}^{0}(n)=\sum_{n=1}^{t} \mathcal{W}_{n} \mathcal{u}_{m}^{0}(n)
$$

$\mathcal{U}^{0}(n)$ represents grain yield in the first $n$ year; $\mathcal{W}_{n}$ $\mathrm{n}$ represents the first $m$ model of the right kind of weight; $\mathcal{U}_{m}^{0}(n)$ represents the results of the $m$ model in the first $n$ years. the combination of model selection using exponentiation, Unitary regression, GM $(1,1)$, These three models. 
7) Exponentiation model is as follows

$$
\delta_{1}^{0}(t)=1593.1 t^{0.3004}
$$

8) Unitary regression model is as follow

$$
\mathcal{S}_{3}^{0}=75.693 x+2209.0
$$

9) $\operatorname{GM}(1,1)$ model is as follows

$$
\mathcal{S}_{3}^{0}=139392.01 \mathrm{e}^{0.018629205 \mathrm{t}-1}-137408 \text { (9) }
$$

10) Bucking and harvesting, processing residues development model is given by the Eq. (10)

$$
\mathcal{D}_{n}=\mathcal{C}_{n} \times \varepsilon+\mathcal{J}_{n} \times \sigma
$$

$\mathcal{D}_{n}$ represents the total bucking and harvesting, processing residues in the first $n$ years; $\mathcal{C}_{n}$ represents the index $n$ of timber harvesting in the first $n$ years; $\varepsilon$ represents felling, bucking residue development coefficient; $\mathcal{J}_{n}$ represents the total amount of wood processing in the first $n$ years; $\sigma$ represents the processing residue development coefficient.

11) Timber harvesting indicators model given by Eq. (11)

$$
\mathcal{C}_{n}=\mathcal{Y}_{n} \times \mathcal{K}
$$

$\mathcal{C}_{n}$ represents the indicators of timber harvesting in the first $n$ years; $\mathcal{Y}_{n}$ represent the current stumpage volume in the first $n$ year; $\mathcal{K}$ represents the ratio of mining to the coefficient.

12) Current stumpage total volume of the model given by $\mathrm{Eq}$. (12)

$$
\mathcal{Y}_{n}=0.984286 n+146055.1
$$

13) The total amount of timber processing model is given by Eq.(13)

$$
\mathcal{J}_{n}=\mathcal{C}_{n}+\mathcal{V}
$$

$\mathcal{C}_{n}$ represents the timber harvesting index in the first $n$ year; $\mathcal{V}$ represents the amount of timber imports..

The template is used to format your $\mathrm{p}$ aper and style the text. All margins, column widths, line spaces, and text fonts are prescribed; please do not alter them. You may note peculiarities. For example, the head margin in this template measures proportionately more than is customary. This measurement and others are deliberate, using specifications that anticipate your paper as one part of the entire proceedings, and not as an independent document. Please do not revise any of the current designations.

\section{SCENARIO SETTING}

\section{A. Demographic change scenario setting}

According to population calculation formula, the future population is mainly affected by the fertility rate. At present, China's population growth rate is about $1.6,{ }^{[10]}$ set it as low level. "National Population Development Report" that future fertility rate should be maintained at about 1.8 , set it as middle level. and set the level of replacement level 2.1 to the high level. Finally, based on the population data in 2014 , the results are as table 1.

TABLE I.

\begin{tabular}{|c|c|c|c|}
\hline \multicolumn{4}{|c|}{ Population in different scenario } \\
\hline & Low & Middle & High \\
\hline 2020year & 13.82 & 13.92 & 14.07 \\
\hline 2030 year & 13.97 & 14.24 & 14.64 \\
\hline 2040 year & 13.64 & 14.05 & 14.7 \\
\hline 2050 year & 13.1 & 13.63 & 14.56 \\
\hline
\end{tabular}

\section{B. food demand scenario setting}

Demand for food by food, feed, food industry, food consumption and seed grain composition. According to Deng Jing (2003), Shi Changliang (2013), Tang Huajun (2012) and "China Statistical Yearbook" (2013), According to Deng Jing (2003), Shi Changliang (2013), Tang Huajun (2012) and "China Statistical Yearbook" (2013) of the data calculated 1978-2012 per capita food demand is in a state of fluctuations, the maximum value of 0.414 tons, the average of 0.374 tons, the minimum value of 0.324 tons, and based on that setting there level of food demand.

\section{Grain yield scenario setting}

The future grain yield is set to be high, medium, low, three kinds of scenarios, Under different scenarios, different forecasting methods will get different weights: Under low situation, the Eq. (7), (8), (9) weight were 60\%, $20 \%, 20 \%$; under medium situation, the weight were $33.3 \%, 33.3 \%, 33.3 \%$; under high situation the weight were $20 \%, 20 \%, 60 \%$.

TABLE II.

\begin{tabular}{|c|c|c|c|}
\hline \multicolumn{4}{|c|}{ Grain yield in different scenario $(\mathrm{kg})$} \\
\hline & Low & Middle & High \\
\hline 2020year & 5543 & 5760 & 6055 \\
\hline 2030 year & 6133 & 6540 & 7050 \\
\hline 2040 year & 6757 & 7398 & 8205 \\
\hline 2050 year & 7431 & 8354 & 9547 \\
\hline
\end{tabular}

D. Biomass energy society development scenario setting

Biomass energy society development scenario is means that: Making ensure food security as a precondition, when the food is not enough, then "returning farmland to forest", hen the food is enough, then "returning forest to farmland" (not taking into account reserve land). Forest basic planning data from China's government commitment and forestry sector for future planning, 2020, 2030, 2040, 2050 year area will be 2.15,2.31,2.46,2.62 million hectares.

\section{RESUlTS AND EVALUATION}

\section{A. Potential and evaluation of biomass energy development under the basic planning}

under the basic plan, in the 2020, 2030, 2040, 2050 biomass energy development potential of $11.62,10.05$, $12.54,13.49$ tons, This is equivalent to 673 million tons, 779 million tons, 840 million, 9.04 tons of standard coal, equivalent to 19.2 percent, 22.2 percent, 23.9 percent in 2012 China's total energy production, if they can all be used, no doubt can effectively alleviate the pressure of energy in our country.

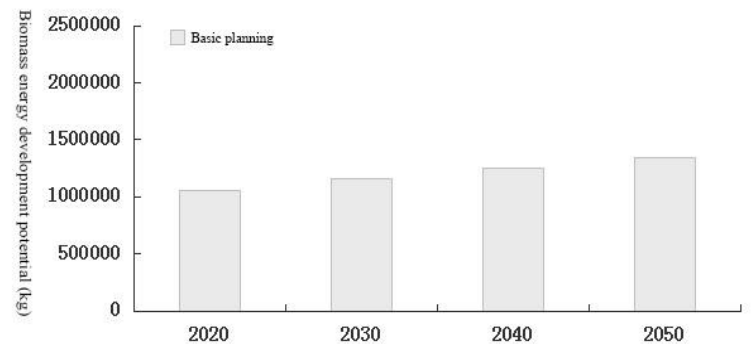

Figure 2. Biomass energy development chart under the basic plan 


\section{B. Potential and evaluation of biomass energy development based on social development}

Taking into account the social factors can be found that Biomass production will increase $2 \%$ to $15 \%$ over the basis for planning, in the long run ; owever, within a short and medium term, due to changes in population, demand, and other factors, Biomass energy development potential will fluctuate up and down, relative to the basic planning, and the maximum amplitude can reach $13 \%$, so in the short term, development of biomass energy can not be too optimistic.

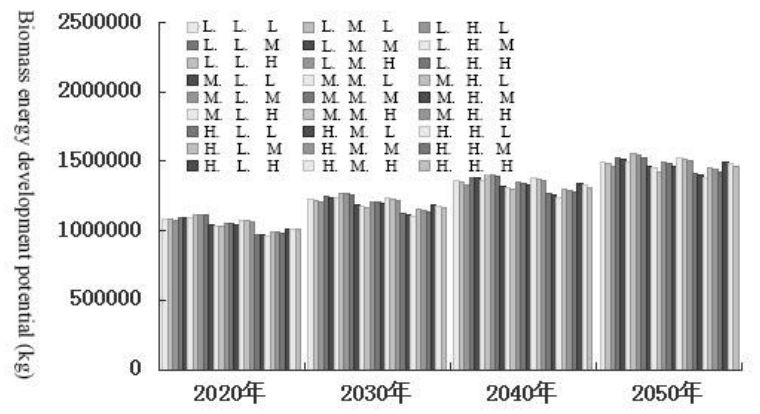

Figure 3. Biomass energy development chart based on social development

\section{Potential and evaluation of biomass energy development in different grain demand}

Under the same conditions, different food demand has great influence on biomass. In low demand, the biomass energy development potential will always keep an increasing trend Relative to the basic planning); In the medium demand, the probability of $1 / 9$ will appear the potential of biomass energy development decline; the probability of 4/9 will appear the potential of biomass energy development decline in the high demand. Especially in the short term, if the demand for food is high, it will inevitably lead to the decline of biological development potential.

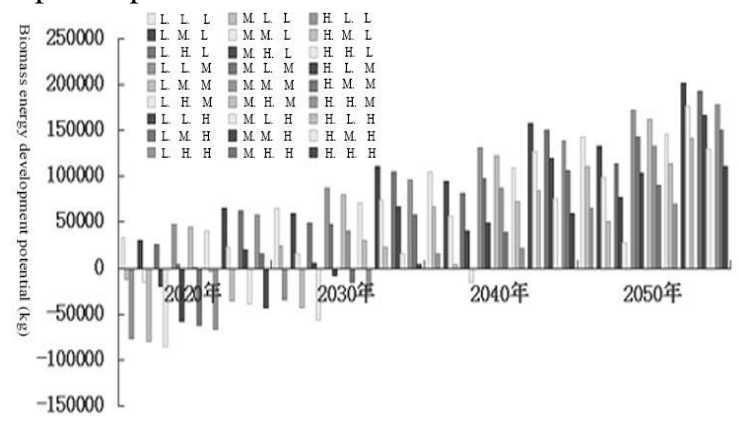

Figure 4. Biomass energy development chart under different grain demand

\section{Potential and evaluation of biomass energy development in different demographic change rate}

Under the same conditions, in the short term, the impact of population growth on the development of biomass energy is not conclusive; in the long term, the different demographic changes have great influence on the development of biomass energy. In the short term, Even under the conditions of low population growth, there may be a potential decline of development potential, under the conditions of high population growth, it may also appear to improve the situation(Relative to basic planning). In the long run, the development potential of biomass energy will keep the trend of increasing, and the impact of the changes in the population is great, low growth rate can produce more than 3.09 tons of high growth potential, equivalent to 2.07 tons of standard coal.

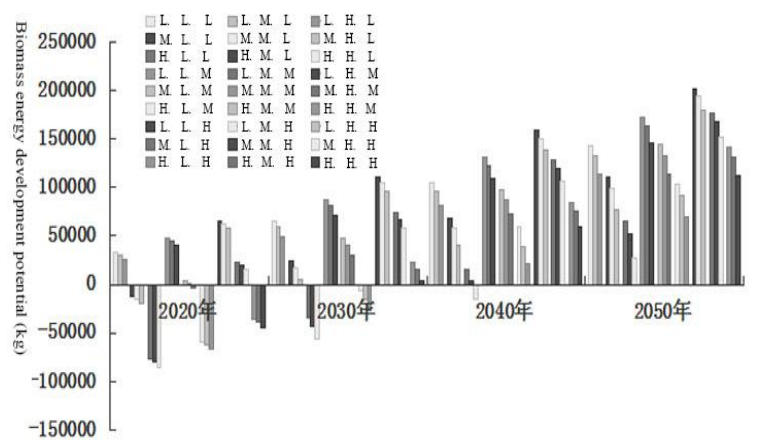

Figure 5. Biomass energy development chart under different population changes

\section{E. Potential and evaluation of biomass energy development under different grain yield}

Grain yields greater influence on biomass energy development potential, especially in the early stage, different yield has great influence on biomass energy potential, the average latent power of different yield can reach $49 \%$; the maximum difference, in the later stage, can be reached $25 \%$.

\section{Conclusions}

Consider the potential of forest biomass energy development from the perspective of social development for the development of forest biomass is significant. Therefore, the research from the current actual situation, and taking into account changes in population growth, demand for food, grain yield and other factors, Using statistical method, scene analysis method, econometric analysis and other methods, from the perspective of social development, to analyze the potential of biomass energy development. It is found that, in general, the development of forest biomass energy in China is huge, and the biggest factor is the food demand, the impact of different population and grain yield on biomass development potential is mainly in the early stage; to the late stage, impact will become small. Therefore, taking these factors into account, for biomass energy development should be from the perspective of a long-term plan, and must control food demand reasonable growth can bring great practical benefit.

\section{ACKNOWLEDGMENT}

The National Natural Science Foundation funded project "Energy security and the environment under the constraint of the total area under agricultural biomass economy mode and compensation mechanism" (71570326)

\section{REFERENCES}

[1] DL Klass,Biomass Renewable Energy Fuels and Chemicals[M]Academic press; SanDiego; USA 1998. 
[2] A Midilli, I Dincer, M Ay.Green energy strategies for sustainable development[J]Energypolicy,2006,(34):3623-3633.

[3] Sun Zhenjun. China's biomass industry and development orientation of the $[\mathrm{J}]$ Agricultural Engineering Journal, 2004,20 (5) 4-5.

[4] Qian Nengzhi, Yin Guopin. The European biomass energy utilization status and experience of foreign [J]. energy, 2007, (12) 10-14.

[5] Lv Wen, Wang Chunfeng. Research on the development of forest biomass energy in China (1) [J]. China energy, 2005,27 (11): 21-26.

[6] Zhou Xiao, Yue Cairong. Research on the development potential of forest biomass energy industry -- Taking Yunnan Province as an example [J] Chinese forestry economy, 2009, (6): 23-26.

[7] National population development strategy research project team. 2007[2015-11-5]. 01/11/content_493677.htm [EB/OL].

[8] China Development Research Foundation. China Development Report 2011/12: population situation and population policy adjustment [M].1. Beijing: China Development Press, 2012

[9] Yi Fuxian, Su Jian. The population of China Outlook 2015-2080-stop family planning urgent EB/OL].2015[2015-115].http://www.chinadmd.com/file/vocuui3so6civxww6sractrs_1.ht ml HTML

[10] Shi Changliang, Jin Yanping. Changes in China's food supply and demand situation: $1978 \sim 2010[\mathrm{~J}]$. economic research reference, 2013, (56): 204-208
[11] Deng Jing. China's food security: An Empirical Analysis of the balance of supply and demand in the food market [J]. China economic issues, 2003, (4) 20-28.

[12] Tang Huajun, Li Zhemin. Study on the per capita grain demand of China's balanced diet pattern [J]. Chinese Agricultural Sciences, 2012,45 (11): 2315-2327.

[13] Lu bu, Chen Yinjun. Analysis and forecasting of the potential of long-term grain yield potential in China [J]. China Agricultural Resources and regionalization, 2005,26 (2): 1-5.

[14] Chen Fangyuan. Application of Grey Combination Model to forecast the output of grain in Hubei province [J]. Journal of Huazhong Agricultural University (SOCIAL SCIENCE EDITION), 2006 (3): 13-16.

[15] Zhu Zaiqing, Chen Fangyuan. The fluctuation of total grain production in Hubei province and its cause of the Journal of Huazhong Agricultural University (SOCIAL SCIENCE EDITION), 2003 (2): 13-16.

[16] Ren Bingxue. The restriction factors and the growth potential of grain production in China [J]. Shijiazhuang municipal Party School Journal, 2009,11 (6): 44-45.

[17] Zhang Yongen, Zhu Qingquan. China's food security situation and Countermeasures in the process of urbanization in China, $[\mathrm{J}] .(3)$ : 270-274.

[18] Zhang Xiliang, Lu Wen. Forest resources in China [M]. Beijing: China Agricultural Press, 2008,17-24. 